\title{
Toward a Canonical Formalism of Non-Perturbative Two-Dimensional Gravity
}

\author{
Tamiaki Yoneya \\ Institute of Physics, University of Tokyo, Meguro-ku, Tokyo 153, Japan
}

Received March 22, 1991

\begin{abstract}
On the basis of the previously proposed action principle describing the theory space of $2 \mathrm{D}$ gravity in less than one-dimension, we develop a systematic canonical formalism for studying the properties of the string equation in the phase space of the cosmological constant and its canonical conjugate, the puncture operator. The string equation is written in a manifestly invariant form under the group of regular canonical transformations in the phase space of generalized coordinate and momentum. As a consequence, the geometrical origin of the generalized Virasoro condition on the partition function (or more precisely, the $\tau$-function) is understood to be the symmetry under the regular area-preserving diffeomorphisms ( $w_{1+\infty}$ symmetry) in the deformed phase space. The deformed canonical formalism can be regarded as a quantization of a classical canonical formalism describing the sphere limit of the theory.
\end{abstract}

\section{Introduction}

The recent development $[1,2]$ of a non-perturbative theory of random surfaces on the basis of the matrix models has provided us a new possibility in studying systems with strongly fluctuating geometries and topologies, without relying upon the intrinsically perturbative world-sheet picture. At present, however, it is not clear whether the matrix models are merely toy models with accidental solvability, or may turn out to exhibit properties of more universal nature which survive in (or are generalized to) realistic quantum gravity and string theories.

It seems therefore important to identify the possible universal framework in which the results of the double scaling limit can be naturally embedded and interpreted. In previous works $[3,4]$, we have proposed a new action principle which describes the structure of the theory space of $2 \mathrm{D}$ gravity, at least, in lessthan-one dimension, and studied its symmetry properties. In particular, the Virasoro condition [5] on the $\tau$-function has been understood as a consequence of a conformal symmetry of the action principle. 
In a previous paper [4], although the explicit derivation has been demonstrated only in the 1-matrix model, it was conjectured that the basic symmetry of the theory is in general a $w_{\infty}$ symmetry, namely, the group of area-preserving diffeomorphisms in the (deformed) phase space of the cosmological constant ${ }^{1}$ and its conjugate, the puncture operator.

Subsequently, there appeared a paper by Fukuma, Kawai, and Nakayama [6] in which they independently arrived at the $w_{1+\infty}$ structure using the formalism of the universal Grassmann manifold to the KP hierarchy in their endeavor for proving a conjecture about the derivation of the higher Virasoro conditions on the $\tau$-functions.

The purpose of the present paper is to generalize the results of the previous works $[3,4]$ by materializing the above conjecture, and to develop a new systematic method for studying the properties of the string equation and the associated $\tau$-function. Our approach is directly developed in the phase space, relying upon the symmetries of the action principle, and does not use the language of the universal Grassmann manifold. Thus our work is complementary to [6], and may hopefully be useful for clarifying the geometrical origin of the $w_{1+\infty}$ structure and trying further generalizations.

In Sect. 2, we fix our notations and reformulate the action principle and its symmetries as a basis toward non-perturbative canonical formalism, emphasizing the notion of the phase space of the cosmological constant and its conjugate. In Sect. 3, we present a general systematic way of solving the variational equation of the action principle by the method of canonical transformations. Then, the $w_{1+\infty}$ symmetry of the string equation is exhibited as a consequence of a gauge symmetry of the action principle which expresses the invariance of the theory under regular canonical transformations. In Sect. 4, we derive the generalized Virasoro condition on the $\tau$-function using the formalism of Sect. 3. Section 5 is devoted to brief discussions on two topics which are relevant to our deformed canonical formalism. The first is the "classical" limit and the second is the "dual" transformation interchanging the generalized coordinate and momentum, which includes as a special case the interchange of $p$ and $q$ in the so-called $(p, q)$ minimal model coupled with gravity. In the final section, we conclude this paper with some remarks.

\section{Action Principle}

\subsection{The Phase Space Representation of the Algebra of psd Operators}

The base space of our canonical formalism is the two-dimensional space of the cosmological constant $x$ and its conjugate $k$. To reproduce the string equation in this phase space, we have to assume that the algebra of functions in the phase space is "deformed."

To any function $f(x, k)$ defined on the phase space which has a Laurent expansion in $k$, we associate a pseudo-differential (psd) operator (or, often called micro-local differential operator) $f$ such that

$$
f(x, k)=\sum f_{i}(x) k^{i} \leftrightarrow f=\sum f_{i}(x) D^{i},
$$

\footnotetext{
${ }^{1}$ In nonunitary models, the terminology "cosmological constant" may not be appropriate. Instead, we should say the phase space of the most relevant coupling constant and its conjugate
} 
where $D=d / d x$. The algebra of psd operators is essentially defined by $D^{i} D^{j}=D^{i+j}$ and $D^{-1} f=\sum_{i=1}^{\infty}(-1)^{i} f^{(i)} D^{-i-1}$. We often use a decomposition of a function $f(x, k)$
given by

$$
\begin{gathered}
f(x, k)=f_{+}(x, k)+f_{-}(x, k), \\
f_{+}(x, k)=\sum_{i \geqq 0} f_{i}(x) k^{i}, \\
f_{-}(x, k)=\sum_{i<0} f_{i}(x) k^{i},
\end{gathered}
$$

and also the operation of taking the adjoint defined by

$$
f \rightarrow f^{*}=\sum(-D)^{i} f_{i}(x) .
$$

The (noncommutative but associative) algebra of the products of functions, $f$ and $g$, denoted as $(f \circ g)(x, k)$ in the phase space, is defined to be isomorphic to the operator algebra of the corresponding psd operators:

$$
\begin{array}{ccc}
(f \circ g)(x, k) & \equiv f(x, k) \exp \left(\check{\partial}_{k} \vec{\partial}_{x}\right) g(x, k) \\
\mathfrak{1} & \uparrow & \uparrow \\
f g & \equiv & \sum_{i, j} f_{i}(x) D^{i} g_{j}(x) D^{j} .
\end{array}
$$

Thus our deformed algebra of functions on the phase space is nothing but a version of the Wigner representation of the algebra of psd operators. The Poisson bracket is defined by $\{f, g\}=f \circ g-g \circ f \leftrightarrow[f, g]=f g-g f$. For example, the cosmological constant $x$ and the conjugate $k$ satisfy $\{k, x\}=1$ which is equivalent to $[D, x]=1$ and means that $k$ is regarded as the eigenvalue of the puncture operator.

The invariant volume integral in the phase is defined to be

$$
\operatorname{Sp} f=\int d x \int d k f(x, k),
$$

where $\int d k \equiv \oint \frac{d k}{2 \pi i}$ with the contour being taken around $k=\infty$, and the $x$-integral is assumed to contain a sufficiently large region of the real axis but be closed in the complex $x$-plane, such that the total derivative with respect to $x$ can always be regarded as zero. It is easy to check that $\operatorname{Sp}(f \circ g)=\mathrm{Sp}(g \circ f)$. Thus the $\mathrm{Sp}$ operation is invariant under arbitrary canonical transformations defined by the above Poisson bracket.

In the following, we mainly use the simpler notations of psd operators and their algebra than geometrically more appealing phase space notations. Since it is completely straightforward to convert between these languages, there should not arise any confusion. The reason why we put emphasis on the phase space formulation here is that assimilation of new canonical formalism developed below may be facilitated by knowing such a geometrical picture behind the abstract psd operator notations. (See also the mathematical literature, e.g., [7] and references therein.)

In Sect. 5, we will discuss the "classical" limit of the formalism, corresponding to the non-deformed Poisson structure, which gives the sphere limit of the theory and may help our understanding of the geometrical structure. 


\subsection{Universal Action Principle}

In this subsection, the "universal" action principle first proposed in [3] and further studied in [4] is presented in a generalized form. The universal action governs the structure of the theory space, in contrast with the $(p, q)$ action $[8,3]$ describing the solution points. The basic ingredients of the dynamics are the bare generalized coordinate $Q_{0}(x, k)$ and the "hamiltonian" $H=H(x, k)=\sum h_{i}(x) k^{i}$. The action is the invariant volume integral of the hamiltonian in the phase space,

$$
\mathscr{A}=\operatorname{Sp} H,
$$

with the constraint,

$$
\left(e^{-H} Q_{0} e^{H}\right)_{-}=0 .
$$

We call $Q=e^{-H} Q_{0} e^{H}$ the dressed generalized coordinate.

At present, the physical interpretation of the condition (2.8) is not clear in our phase space language. But as a fact, it requires that the dressed generalized coordinate $Q$ must be regular at $k=0$. Presumably this should be interpreted as a sort of smoothness requirement at short distances. Note that $k$ is essentially the measure of the volume of the surfaces.

The action (2.7) can be motivated from the matrix model [9] before taking the double scaling limit. The canonical transformation $e^{H}$ corresponds to the change of an arbitrary fixed basis of polynomial wave functions for the $N$-fermion systems, describing the matrix model of finite $N$, to the basis formed by the orthonormal polynomials with respect to the potential. The partition function of the matrix model is then given by the squared determinant of the transformation matrix. Thus the action (2.7) corresponds to the effective action of the matrix model apart from a factor 2 .

Now to take into account the constraint, we introduce a lagrange multiplier, $P(x, k) \equiv P_{+}(x, k)$ and formulate the action principle as

$$
\mathscr{A}=\operatorname{Sp}\left(P e^{-H} Q_{0} e^{H}-H\right) .
$$

The dynamical variables which are subject to variation are the Laurent coefficients of the functions $H$ and $P$, while the choice of the generalized coordinate $Q_{0}$ specifies the subspace of the theory space of $2 \mathrm{D}$ gravity. For example, the choice $Q_{0}=k^{2}$ corresponds to the one-matrix model, and the higher power of $k$ to two-(and more)matrix models.

The variational equations of the action (2.9) are

$$
\begin{gathered}
{[P, Q]=1,} \\
Q=e^{-H} Q_{0} e^{H}, \\
Q_{-}=0 .
\end{gathered}
$$

Thus the lagrange multiplier $P$ is nothing but the dressed generalized momentum which is canonically conjugate to $Q$. The equivalence of these equations with the string equations for the appropriate choices of the bare generalized coordinate will be proven in the next section.

In the rest of the present section, we discuss the symmetry properties of the action principle. There exist two kinds of symmetries which are both of infinite dimension. In the following we denote $e^{-H}$ by $K$ for notational simplicity. 
(i) Global KP-flow symmetry:

$$
\begin{gathered}
\delta_{\mathrm{KP}} K=-\left(K \varepsilon(D) K^{-1}\right)_{-} K, \\
\delta_{\mathrm{KP}} P=\left[\left(K \varepsilon(D) K^{-1}\right)_{+}, P\right],
\end{gathered}
$$

where $\varepsilon(D)\left[=\varepsilon(D)_{+}\right]$is an arbitrary differential operator with constant coefficients, $\varepsilon(D)=\sum_{i=1}^{\infty} \varepsilon_{i} D^{i}$.

(ii) Canonical gauge symmetry:

$$
\begin{aligned}
& \delta_{c} K=-G K, \\
& \delta_{c} P=[P, G] .
\end{aligned}
$$

where $G\left(=G_{+}\right)$is an arbitrary differential operator, $G=\sum_{i \geqq 0} g_{i}(x) D^{i}$.

Let us discuss the consequences of these symmetries. Firstly, by using the canonical gauge symmetry (ii), we can eliminate the positive power part of the hamiltonian and hence reduce the form of $H$ to $H=H_{-}=\sum_{i<0} h_{i}(x) D^{i}$. This is because any psd operator of positive order can be decomposed into the form $\left(1+\sum_{i \geqq 1} a_{i} D^{i}\right) \exp \sum_{i \geqq 1} b_{i} D^{-i}$. This is proven by first considering a psd operator $F=\sum_{i \leqq N} f_{i} D^{i}$ finite order $N$. We can recursively determine the coefficients $a_{i}$ and $b_{i}$ in terms of $f_{i}$. Then let $N \rightarrow \infty$. Thus we can write $\exp -H=\left(1+A_{+}\right) \exp -\tilde{H}$ with $A_{+}=\sum_{i \geqq 1} a_{i} D^{i}, \quad \widetilde{H}=\widetilde{H}_{-}$. Making a finite canonical transformation $P \rightarrow\left(1+A_{+}\right) P\left(1+A_{+}\right)^{-1}$ and using $\operatorname{Sp} H=\operatorname{Sp} \tilde{H}$ we obtain

$$
\mathscr{A}=\operatorname{Sp}\left(P K Q_{0} K^{-1}+\log K\right)
$$

where $K=\exp -\tilde{H}$.

Furthermore, when $Q_{0}$ is assumed to be of finite positive order $(=q)$ with constant highest order coefficient, $Q_{0}$ can be reduced to a monomial differential operator $D^{q}$, since there is a psd $J$ of the form $\sum_{i \geqq 0} j_{i}(x) D^{-i}$ such that $Q_{0}=\exp (-J) D^{q} \exp J$, apart from an overall normalization. The psd operator $J$ can be absorbed into a redefinition of $\tilde{H}$ which only changes the action by a nondynamical constant. Unless otherwise stated, we will assume below that the above redefinition is performed so that $H=H_{-}$and $d Q_{0} / d x=0$.

A more important implication of the canonical gauge symmetry is that the variational equations are invariant under arbitrary regular canonical transformations. Here by regular, we mean that they are generated by differential operators $G=G_{+}$,

$$
\delta_{c}[P, Q]=0, \quad\left(\delta_{c} P\right)_{-} \equiv([P, G])_{-}=0, \quad\left(\delta_{c} Q\right)_{-} \equiv([Q, G])_{-}=0 .
$$

In particular, we can choose the set $\left\{w_{n}^{(i)} \equiv-Q^{n+i-1} P^{i-1} ; i \geqq 1, n+i-1 \geqq 0\right\}$ as a basis for $G$,

$$
G=\sum_{i=1, n+i-1 \geqq 0}^{\infty} \varepsilon_{n i} w_{n}^{(i)}
$$


The $w_{n}^{(i)}$ 's constitute a quantized version of the closed algebra [10] of the regular area-preserving diffeomorphisms in the phase space of the generalized coordinate and momentum (without central extension),

$$
\left[w_{m}^{(i)}, w_{n}^{(j)}\right]=((j-1) m-(i-1) n) w_{m+n}^{(i+j-2)}+\ldots,
$$

where (...) indicates the lower order terms corresponding to the quantum effect. We call this algebra $w_{1+\infty}$ algebra since it includes the case $i=1$ [11]. The conformal transformation $Q \rightarrow Q+\varepsilon_{n} Q^{n+1}$, from which the Virasoro condition on the $\tau$-function is derived, as discussed in the previous paper [4], is a special case of (2.19) with $i=2, G=-\sum_{n=-1}^{\infty} \varepsilon_{n} Q^{n+1} P$.

Now let us turn to the global KP-flow symmetry. Since the variational equations are invariant under the flow by the evolution equations of the KP hierarchy, the solution space can be parametrized by an infinite number of the KP time parameters $x_{r}(r \geqq 2)$, such that

$$
\begin{gathered}
\frac{\partial Q}{\partial x_{r}}=\left[L_{r}, Q\right], \\
\frac{\partial P}{\partial x_{r}}=\left[L_{r}, P\right], \\
L_{r} \equiv\left(K D^{r} K^{-1}\right)_{+} .
\end{gathered}
$$

Here in reducing the equation for $Q$ to the form (2.21), we have assumed that the generalized coordinate $Q_{0}$ is a psd operator with constant coefficients. In later discussions, we will mainly assume this for simplicity.

The most important consequence of the KP-flow symmetry is that there exists a function, called the $\tau$-function [12], associated with the eigenfunction of the dressed generalized coordinate $Q$. Namely, the action of the "wave operator" $K=\mathrm{epx}-\widetilde{H}$ on a "plane" wave function can be represented in the following form,

$$
\begin{gathered}
K \exp \sum_{r=0}^{\infty} x_{r} k^{r}=\frac{V(k) \tau(x)}{\tau(x)}, \\
K^{*-1} \exp -\sum_{r=0}^{\infty} x_{r} k^{r}=\frac{V^{\dagger}(k) \tau(x)}{\tau(x)}, \\
V(k) \equiv \exp \left(\sum_{r=0}^{\infty} x_{r} k^{r}\right) k^{\partial_{0}} \exp \left(-\sum_{r=1}^{\infty} \frac{k^{-r}}{r} \partial_{r}\right) \\
=: \exp \phi(k):, \\
V^{\dagger}(k)=: \exp -\phi(k): .
\end{gathered}
$$

Here we have introduced an auxiliary zero-mode variable $x_{0}$ with the assumption that $\partial_{0} \tau=0$, and defined a field operator $\phi(k)$ in the $k$-space,

$$
\begin{gathered}
\phi(k) \equiv x_{0}+\alpha_{0} \log k-\sum_{r \neq 0} \frac{k^{-r}}{r} \alpha_{r}, \\
\alpha_{r} \equiv \partial_{r} \quad(r \geqq 0), \\
\alpha_{-r} \equiv r x_{r} \quad(r>0) .
\end{gathered}
$$


The KP-flow equations $\partial_{r} K=-\left(K D^{r} K^{-1}\right)_{-} K(r \geqq 2)$ are then equivalent to Hirota's bilinear equation (see [12]) for the $\tau$-function,

$$
\int d k(V(k) \tau(x))\left(V^{\dagger}(k) \tau\left(x^{\prime}\right)\right)=0,
$$

where $\left\{x_{r}\right\}$ and $\left\{x_{r}^{\prime}\right\}$ are two arbitrary sets of the KP time parameters.

The importance of the $\tau$-function lies in the fact that it is interpreted as the partition function of $2 \mathrm{D}$ gravity. In terms of the matrix model, the $\tau$-function is known to be the square root of the partition function. This is consistent with the meaning of the operator $K$ in the matrix model as explained in the beginning of this subsection. For a related discussion of the $\tau$-function from a different viewpoint, see [13]. As in the previous paper [4], the bilinear equation will play crucial roles in our discussions on the symmetry properties of the $\tau$-function in later sections.

\section{Bilinear String Equation}

\subsection{Solving the Variational Equation by Canonical Transformation}

In this section, we discuss the general solution to the variational equations (2.10) (2.12) of the action principle in the case where $Q_{0}$ is a general psd operator with constant coefficients,

$$
Q_{0}=\alpha(D) \text {. }
$$

We assume that $\alpha(D)$ is invertible as $D=D\left(Q_{0}\right)$.

Let us start from considering the general solution for the "bare" momentum $P_{0} \equiv K^{-1} P K$ satisfying,

$$
\left[P_{0}, Q_{0}\right]=1
$$

It is given by

$$
P_{0}=-\frac{1}{2}\left(\frac{1}{\alpha^{\prime}(D)} x+x \frac{1}{\alpha^{\prime}(D)}\right)+\beta(D)
$$

where $\alpha^{\prime}(D)=\frac{d}{d D} \alpha(D)$ and $\beta(D)$ is an arbitrary psd operator with constant coefficients and, for later convenience, we have chosen the symmetric ordering for the first term. This shows that the most general solution for the dressed momentum must take the form,

$$
P=K P_{0} K^{-1}
$$

with the condition,

$$
P_{-}=\left(K P_{0} K^{-1}\right)_{-}=0 .
$$

From the discussion of the previous section, we can parametrize the constant operator $\beta(D)$ in terms of an infinite number of the time variables (namely, the coupling constants) of the KP flow symmetry, which are denoted by $x_{r}(r \geqq 2$, 
$\left.x_{1} \equiv x\right)$. This is done by requiring that

$$
\begin{gathered}
\frac{\partial P_{+}}{\partial x_{r}}=\left[\left(K D^{r} K^{-1}\right)_{+}, P_{+}\right], \\
\frac{\partial P_{-}}{\partial x_{r}}=0,
\end{gathered}
$$

for $\partial_{r} K=-\left(K D^{r} K^{-1}\right)_{-} K$. After some calculations, (3.6) leads to

$$
\left(K \frac{\partial \beta(D)_{+}}{\partial x_{r}} K^{-1}\right)_{+}=\left[P_{+}, K D^{r} K^{-1}\right]_{+} .
$$

Using the inversion of the function $\alpha(D)$ and the commutation relation $[P, Q]=1$, this is reduced to

$$
\left(K \frac{\partial \beta(D)_{+}}{\partial x_{r}} K^{-1}\right)_{+}=-r\left(K D^{r-1} D^{\prime}\left(Q_{0}\right) K^{-1}\right)_{+} \cdot
$$

Thus, choosing the origins for the variables $x_{r}$ 's appropriately, we can set

$$
\beta(D)_{+}=-\sum_{r=q}^{\infty} r x_{r}\left(D^{r-1} D^{\prime}\left(Q_{0}\right)\right)_{+},
$$

where $q$ is the order of $Q_{0}$. Note that the order of $D^{r-1} D^{\prime}\left(Q_{0}\right)$ is $r-q$ which determines the range of the summation over $r$. Similarly, (3.7) leads to

$$
\left(K \frac{\partial \beta(D)}{\partial x_{r}} K^{-1}\right)_{-}=-r\left(K D^{r-1} \frac{1}{\alpha^{\prime}(D)} K^{-1}\right)_{-} .
$$

Since $\alpha^{\prime}(D) D^{\prime}\left(Q_{0}\right)=1,(3.9)$ and (3.11) are mutually consistent and show that we can generally set

$$
\beta(D)=-\sum_{r=2}^{\infty} r x_{r} D^{r-1} \alpha^{\prime}(D)^{-1},
$$

apart from the ambiguity of adding the negative power part of a constant psd operator of the form $\sum_{s<0} c_{s} D^{s} \alpha^{\prime}(D)^{-1}\left(=\sum_{s \leqq-q} c_{s-1+q} D^{s} / q\right.$ for the case $\left.\alpha(D)=D^{q}\right)$. This ambiguity can be eliminated by a redefinition of the operator $K$, utilizing the degree of freedom of right multiplication of an arbitrary operator with constant coefficients to $K$, which does not affect the action principle. Indeed, an infinitesimal redefinition $K \rightarrow K\left(1+\varepsilon_{0} \log D+\sum_{r=1}^{\infty} \varepsilon_{-r} D^{-r}\right)$ induces the change $\delta P_{0}=\varepsilon_{0} D^{-1} \alpha^{\prime}(D)^{-1}+\sum_{r=1}^{\infty} r \varepsilon_{-r} D^{-r-1} \alpha^{\prime}(D)^{-1}$. Although the contribution of the logarithmic term of the constant operator in the right multiplication does induce a non-integrable change of the action, it only affects the ambiguity of adding a constant to the action and a change of the normalization of the wave function.

We found that the general solution to the bare generalized momentum parametrized by the KP flow is given by

$$
P_{0}=-\frac{1}{2} \sum_{r=1}^{\infty} r\left(x_{r} \alpha^{\prime}(D)^{-1} D^{r-1}+\alpha^{\prime}(D) D^{r-1} x_{r}\right),
$$


up to the addition of an irrelevant constant psd operator. The dressed generalized momentum and coordinate are $(q \geqq 2)$

$$
\begin{gathered}
P=K P_{0} K^{-1}=-\sum_{r=q}^{\infty} r x_{r}\left(K D^{r-1} \alpha^{\prime}(D)^{-1} K^{-1}\right)_{+}, \\
Q=K Q_{0} K^{-1}=\left(K \alpha(D) K^{-1}\right)_{+},
\end{gathered}
$$

with the conditions

$$
\begin{aligned}
& P_{-}=\left(K P_{0} K^{-1}\right)_{-}=0, \\
& Q_{-}=\left(K Q_{0} K^{-1}\right)_{-}=0 .
\end{aligned}
$$

For $\alpha(D)=D^{q},(3.14)$ gives

$$
P=-\frac{1}{q} \sum_{i=0}^{\infty}(i+q) x_{i+q}\left(Q^{\frac{i}{q}}\right)_{+}
$$

which is the form first proposed by Douglas [2]. We emphasize that although (3.5) implies an infinite number of differential equations, they are equivalent with $[P, Q]=1$ which contains $q-1$ differential equations for $q-1$ independent coefficients of the operator $K$ satisfying $Q_{-}=0$. The origin of the extra differential equations is the $w_{1+\infty}$ symmetry as a consequence of the canonical gauge symmetry.

What we have shown is that the string equation as the variational equation to our action principle is reduced to two operator conditions (3.16) and (3.17), which are manifestly invariant under the general regular canonical gauge transformations (2.19). Since an arbitrary polynomial in $P$ and $Q$ satisfies the same condition as a consequence of these two conditions, it is convenient to express this situation in the following form:

$$
\left(K \mathscr{P}\left(Q_{0}, P_{0}\right) K^{-1}\right)_{-}=0,
$$

for any polynomial $\mathscr{P}$ of $Q_{0}$ and $P_{0}$.

\subsection{Bilinear Form of the String Equation}

Let us now rewrite the string equation as the condition on the $\tau$-function, i.e., the partition function of $2 \mathrm{D}$ gravity. The operator equations of the type (3.19) can in general be rewritten as the bilinear conditions on the $\tau$-function, using the formula

$$
\left(P Q^{*}\right)_{-n}=(-1)^{n} \int d k\left(P e^{x k}\right) \partial_{x}^{n-1}\left(Q e^{-x k}\right) \quad(n \geqq 1),
$$

which was discussed in the appendix of the previous paper [4] following [12]. This formula is valid for any two psd operator $P$ and $Q$.

For arbitrary two polynomials satisfying $\mathscr{P}=\mathscr{P}_{1} \mathscr{P}_{2},(3.19)$ is thus equivalent to

$$
\int d k\left(K \mathscr{P}_{1}\left(Q_{0}, P_{0}\right) e^{x k}\right)\left(K^{*-1} \mathscr{P}_{2}\left(Q_{0}, P_{0}\right)^{*} e^{-x^{\prime} k}\right)=0 .
$$

Furthermore, since the solution is parametrized by the KP-flow equation, the above equation is actually generalized to

$$
\int d k\left(K \mathscr{P}_{1} e^{\xi(x, k)}\right)\left(K^{*+1} \mathscr{P}_{2}^{*} e^{\xi\left(x^{\prime}, k\right)}\right)=0,
$$


where $\xi(x, k)=\sum_{r=0}^{\infty} x_{r} k^{r}$ and $\xi\left(x^{\prime}, k\right)=\sum_{r=0}^{\infty} x_{r}^{\prime} k^{r}$. This is because the KP equations
imply

$$
\frac{\partial}{\partial x_{r}} K \mathscr{P}_{1}\left(Q_{0}, P_{0}\right) e^{\xi(x, k)}=\left(K D^{r} K^{-1}\right)_{+} K \mathscr{P}_{1}\left(Q_{0}, P_{0}\right) e^{\xi(x, k)},
$$

and hence,

$$
\int d k\left(\partial_{1}^{l_{1}} \ldots \partial_{n}^{l_{n}} K \mathscr{P}_{1} e^{\xi(x, k)}\right)\left(K^{*-1} \mathscr{P}_{2}^{*} e^{\xi(x, k)}\right)=0,
$$

which proves (3.22). Then in terms of the $\tau$-function, this equation reads

$$
\int d k\left(\widetilde{\mathscr{P}}_{1}\left(P_{0}, Q_{0}\right) V(k) \tau(x)\right)\left(\widetilde{\mathscr{P}}_{2}\left(P_{0}, Q_{0}\right)^{*} V^{\dagger}(k) \tau\left(x^{\prime}\right)\right)=0,
$$

where $Q_{0}(k), P_{0}(k), Q_{0}^{*}(k), P_{0}^{*}(k)$, defined by, respectively,

$$
\begin{gathered}
Q_{0} e^{\xi}=Q_{0}(k) e^{\xi}, \quad P_{0} e^{\xi}=P_{0}(k) e^{\xi}, \\
Q_{0}^{*} e^{-\xi}=Q_{0}(k)^{*} e^{-\xi}, \quad P_{0}^{*} e^{-\xi}=P_{0}(k) e^{-\xi},
\end{gathered}
$$

are independent of the parameters $x_{r}^{\prime}$ 's and are given by

$$
\begin{gathered}
Q_{0}(k)=\alpha(k)=Q_{0}(k)^{*}, \\
P_{0}(k)=-\left(\alpha^{\prime}(k)^{-1} \frac{d}{d k}-\frac{\alpha^{\prime \prime}(k)}{2 \alpha^{\prime}(k)^{2}}\right)=-P_{0}(k)^{*} .
\end{gathered}
$$

In (3.25), the tilde over $\mathscr{P}_{1}, \mathscr{P}_{2}$ indicates that the orderings of the operators $Q_{0}, P_{0}$ are reversed from the original ones of $\mathscr{P}_{1}, \mathscr{P}_{2}$. These are the most general bilinear forms of the string equation and contain the whole information on the partition function of 2D gravity coupled with $(p, q)$ matter fields. In the previous paper [4], some special cases of them are utilized in a more intricate way.

\section{Canonical Symmetry of the Partition Function}

In this section, as a first application of our canonical formalism, we derive the linear conditions, which is equivalent with the Schwinger-Dyson equation, on the $\tau$ function coming from the canonical gauge symmetry, generalizing a result of the previous paper [4].

In the representation (3.25) of the string equation, the canonical gauge transformation generated by (2.19) can be interpreted to act on the $\tau$-function as

$$
\begin{gathered}
\tau(x) \rightarrow \tau(x)+\delta_{c} \tau(x), \\
\delta_{c} \tau(x)=\sum_{n \geqq-i+1, i \geqq 1} \varepsilon_{n i} \oint \frac{d k}{2 \pi i} N\left(V^{\dagger}(k) w_{n}^{(i)}(k) V(k)\right) \tau(x), \\
w_{n}^{(i)}(k)=P_{0}(k)^{i-1} Q_{0}(k)^{n+i-1} \quad(i \geqq 1, n+i-1 \geqq 0) .
\end{gathered}
$$

Here the normal symbol $N(\ldots)$ is defined with respect to the short-distance singularity of the product of the vertex operators:

$$
N\left(V^{\dagger}(k) \mathcal{O}(k) V(k)\right)=\lim _{u \rightarrow k}\left(V^{\dagger}(u) \mathcal{O}(k) V(k)-\mathcal{O}(k) \frac{1}{u-k}\right) .
$$

The generators (4.3) satisfy the $w_{1+\infty}$ algebra (2.20). 
Let us first prove the above statement. Using the commutation relations, ${ }^{2}$

$$
\begin{gathered}
{\left[W_{n}^{(i)}, V(k)\right]=w_{n}^{(i)}(k) V(k),} \\
{\left[W_{n}^{(i)}, V^{\dagger}(k)\right]=-w_{n}^{(i)}(k)^{*} V^{\dagger}(k),}
\end{gathered}
$$

where

$$
W_{n}^{(i)} \equiv \oint \frac{d k}{2 \pi i} N\left(V^{\dagger}(k) w_{n}^{(i)}(k) V(k)\right),
$$

and the relations between the operator equations (3.16), (3.17), and the bilinear equations for the $\tau$-function as discussed in the previous section, it is easy to see that the transformation (4.2) induces the following change of the string equation:

$$
\delta_{c}\left(K \mathscr{P}\left(Q_{0}, P_{0}\right) K^{-1}\right)_{-}=[G, \mathscr{P}(Q, P)]_{-}=0,
$$

where $G$ is given by (2.19) and in deriving the first equality we have used the string equation $\left(K \mathscr{P}\left(Q_{0}, P_{0}\right) K^{-1}\right)_{-}=0$ for the polynomial $\mathscr{P}$. Thus, comparing $(4.8)$ with (2.18), the action of the canonical gauge transformation to the $\tau$-function is given by (4.2) in the space of the solutions of the variational equation, as claimed above.

The result (4.8) shows that if there exists a general solution to the $\tau$-function, then $\tau+\delta_{c} \tau$ also satisfies the same property. Next we prove that this is actually equivalent to a stronger statement that

$$
W_{n}^{(i)} \tau=0 \quad(n \geqq-i+1, i \geqq 1) .
$$

For $i=1,(4.9)$ is obviously satisfied since they are nothing but the reduction conditions of the KP hierarchy which are equivalent to the constraint $\left(K Q_{0} K^{-1}\right)_{-}=0$ in the action principle. For example, if $Q_{0}=k^{q}$,

$$
\begin{aligned}
W_{n}^{(1)} & =\oint \frac{d k}{2 \pi i} N\left(V^{\dagger}(k) k^{n q} V(k)\right) \\
& =-\oint \frac{d k}{2 \pi i} k^{n q} \frac{d \phi(k)}{d k} \\
& =\partial_{n q} .
\end{aligned}
$$

For $i=2,(4.7)$ are the Virasoro generators. In particular,

$$
\begin{aligned}
W_{-1}^{(2)} & =-\oint \frac{d k}{2 \pi i} N\left(V^{\dagger}(k)\left(\alpha^{\prime}(k)^{-1} \frac{d}{d k}-\frac{\alpha^{\prime \prime}(k)}{2 \alpha^{\prime}(k)^{2}}\right) V(k)\right) \\
& =\frac{1}{2} \oint \frac{d k}{2 \pi i} \alpha^{\prime}(k)^{-1}:\left(\frac{d \phi(k)}{d k}\right)^{2}: .
\end{aligned}
$$

On the other hand, using the KP equation in the component form $\partial_{r} K$ $=-\left(K D^{r} K^{-1}\right)_{-} K$, we find that the string equation $\left(K P_{0} K^{-1}\right)_{-}=0$ is equivalent to

$$
\left(W_{-1}^{(2)} \tau\right) V(k) \tau-\tau\left(V(k) W_{-1}^{(2)} \tau\right)=0 .
$$

It is easy to show, using an inductive argument with respect to the pole residues in $k$, that the general solution of (4.15) is

$$
W_{-1}^{(2)} \tau=c \tau,
$$

${ }^{2}$ Note that the vertex operators are the bosonized forms of the free fermions 
where $c$ is a numerical constant independent of $x_{r}(r \geqq 1)$. Combining this property and the canonical gauge symmetry of the $\tau$-function, namely, that $\tau+\delta_{c} \tau$ also satisfies (4.15), we have $\left[W_{-1}^{(2)}, W_{n}^{(i)}\right] \tau=(n+i-1) W_{n-1}^{(i)} \tau=0$ which proves (4.9).

In [6], the $w_{1+\infty}$ condition on the $\tau$-function has been derived by using the universal Grassmannian representation for the $\tau$-function. The merits of our derivation based upon the action principle are that it clarifies the geometrical origin of the condition and, hopefully, that without using directly the Grassmannian language, it might be generalized to 2D gravity with more general matter systems than minimal conformal models and the associated massive theories. Since a detailed discussion about the further reduction of the $w_{1+\infty}$ condition to the $w_{q}$ condition in the case $\alpha(D)=D^{q}$ has already been given in [6], we do not elaborate in that direction in the present paper.

\section{Further Applications}

\subsection{Classical Limit}

As we have emphasized in Sect. 2, the algebra of functions in the phase space of our canonical formalism is deformed. To clarify the nature of the deformation, it is useful to introduce explicitly the deformation parameter which we denote $\lambda$. The correspondence with the algebra of psd operators now becomes as follows:

$$
\begin{array}{cc}
(f \circ g)(x, k) & \equiv f(x, k) \exp \left(\lambda{\overleftarrow{\partial_{k}}}_{\vec{\partial}}\right) g(x, k) \\
\uparrow & \downarrow \\
f g & \equiv \sum_{i, j} f_{i}(x)(\lambda D)^{i} g_{j}(x)(\lambda D)^{j} .
\end{array}
$$

With this redefinition, all of the foregoing formalism are valid as they stand. In this subsection, the product symbol $\circ$ should always be understood in the above sense. The Poisson bracket is defined by

$$
\{f, g\}=\frac{1}{\lambda}(f \circ g-g \circ f) .
$$

Thus a finite canonical transformation of a function $f$ generated by $H$ is $f \rightarrow \exp \left(-\frac{H \circ}{\lambda}\right) f \exp \left(\frac{\circ H}{\lambda}\right)$, where $\exp \left(-\frac{H \circ}{\lambda}\right)=1-\frac{H \circ}{\lambda}+\frac{1}{2}\left(\frac{H \circ}{\lambda}\right)^{2}+\ldots$ The string equation is now $\{P, Q\}=1$.

The limit $\lambda \rightarrow 0$ defines the classical phase space in which $\{f, g\}_{\lambda=0}$ $=\frac{\partial f}{\partial k} \frac{\partial g}{\partial x}-\frac{\partial f}{\partial x} \frac{\partial g}{\partial k}$ and a finite canonical transformation is given by $\lim _{\lambda \rightarrow 0} \exp (-H \circ / \lambda) f \exp (\circ H / \lambda)=f-\{H, f\}_{\lambda=0}+(1 / 2)\{H,\{H, f\}\}_{\lambda=0}+\ldots$. Then we naturally come to a question of what is the classical theory of the action principle. We shall demonstrate that it describes the 2D quantum gravity on the sphere, as it should be.

Let us study the operator string equation with $\alpha(k)=k^{q}$ and $K=\exp -H \circ / \lambda$,

$$
\left(K P_{0} K^{-1}\right)_{-}=\left(K Q_{0} K^{-1}\right)_{-}=0 \text {. }
$$


Taking the limit $\lambda \rightarrow 0$, we obtain

$$
\begin{gathered}
\left((x+E(x, k))(k+F(x, k))^{1-q}+\sum_{r=2}^{\infty} r x_{r}(k+F(x, k))^{r-q}\right)_{-}=0, \\
(k+F(x, k))_{-}^{q}=0 .
\end{gathered}
$$

Here we defined the functions $E$ and $F$, expressing the effects of the classical canonical transformation,

$$
\begin{aligned}
& k+F(x, k)=\lim _{\lambda \rightarrow 0} \exp \left(-\frac{H \circ}{\lambda}\right) k \exp \left(\frac{\circ H}{\lambda}\right), \\
& x+E(x, k)=\lim _{\lambda \rightarrow 0} \exp \left(-\frac{H \circ}{\lambda}\right) x \exp \left(\frac{\circ H}{\lambda}\right) .
\end{aligned}
$$

We can expand them as $F(x, k)=\sum_{i=1}^{\infty} f_{-i}(x) k^{-i}, E(x, k)=\sum_{i=2}^{\infty} e_{-i}(x) k^{-i}$.

To avoid inessential complications we specialize to the case $q=2$. The solution to the constraint equation (5.4) is then easily found to be

$$
F=k\left(\left(1+f_{-1} k^{-2}\right)^{1 / 2}-1\right) \text {. }
$$

Substituting (5.7) into (5.3) and taking the residue with respect to $k$, we obtain the equation for $f_{-1}$,

$$
\sum_{n=0}^{\infty}(2 n+1) x_{2 n+1}\left(2 f_{-1}\right)^{n} \frac{\Gamma\left(\frac{2 n+1}{2}\right)}{n ! \Gamma(1 / 2)}=0 .
$$

Since $f_{-1}=d h_{-1} / d x$, the second derivative of the free energy, this is nothing but the Landau-Ginzburg equation derived from the one-matrix model in the large $N$-limit. Vanishing of the higher residue terms in (5.3) is guaranteed by the canonical structure of the formalism. It is not difficult to check this explicitly. For instance, the $k^{-2}$ term of (5.3) reads

$$
-(1 / 2) \sum_{n=0}^{\infty}(2 n+1) x_{2 n+1}\left(2 f_{-1}\right)^{n+1} \frac{\Gamma\left(\frac{2 n+1}{2}\right)}{(n+1) ! \Gamma(1 / 2)}+h_{-1}=0,
$$

which is derived from (5.8) by multiplying $d f_{-1} / d x$ and integrating with respect to $x$. Similarly, for general $q$, the first $q-1$ coefficients of the classical string equation, $\lim _{\lambda \rightarrow 0}\left(K \circ P_{0} \circ K^{-1}\right)_{-}=0$, give the Landau-Ginzburg equations after solving the constraint equations, and the vanishing of the higher coefficients are the consequences of them.

The fact that the sphere limit is formulated as the classical action formalism corresponding to our deformed canonical formalism is interesting from the viewpoint of constructing a general framework for nonperturbative string theory. This suggests an entirely new possibility of setting up the quantum theory of random geometry with the fluctuations of topology, other than the string field theory and its quantization. In [4], we have explored a different possibility of quantizing directly the Landau-Ginzburg theory by regarding $f_{-1}$ as the 
dynamical variable to be quantized. However, it turned out that such an approach led to an extremely complicated representation of the partition function, in sharp contrast with 1D gravity, i.e., particle theory [14].

\subsection{Dual Transformation}

In solving the variational equations we developed a nonsymmetrical treatment of generalized coordinate and momentum, in spite of the fact that the equations $\left(K P_{0} K^{-1}\right)_{-}=\left(K Q_{0} K^{-1}\right)_{-}=0$ are manifestly symmetric with respect to the coordinate and momentum. In our formalism, it is however straightforward to interchange the roles of the coordinate and momentum by a canonical transformation, which we refer to as "dual" transformation. ${ }^{3}$ In this final subsection, we briefly discuss the properties of the dual transformation.

Let us take the massive $(p, q)$ theory described by

$$
\begin{gathered}
P_{0}=\frac{1}{2 q} \sum_{i=-q+1}^{p}(i+q)\left(x_{i+q} D^{i}+D^{i} x_{i+q}\right), \\
Q_{0}=D^{q},
\end{gathered}
$$

and consider transforming them to the $(q, p)$ theory described by interchanging the representations of $P_{0}$ and $Q_{0}$ such that

$$
\begin{gathered}
\tilde{P}_{0}=-\frac{1}{2 p} \sum_{i=-p+1}^{q}(i+p)\left(\tilde{x}_{i+p} D^{i}+D^{i} \tilde{x}_{i+p}\right), \\
\tilde{Q}_{0}=D^{p} .
\end{gathered}
$$

We set $x_{p+q}=\frac{2 q}{p+q}, \tilde{x}_{p+q}=-\frac{2 p}{p+q}$ for notational simplicity. From the general discussion of Sect. 3.1, it is guaranteed that there exists a transformation $U$ satisfying

$$
\begin{aligned}
& P_{0}=U \widetilde{Q}_{0} U^{-1}, \\
& Q_{0}=U \widetilde{P}_{0} U^{-1},
\end{aligned}
$$

in the form

$$
U=\sum_{i=0}^{\infty} u_{-i} D^{-i}
$$

Note that the operator $U$ is not analytic with respect to $P_{0}$ and $Q_{0}$, and, hence, that it in general does change the $\tau$-function.

The operator $U$ is unique apart from a normalization factor which is independent of $x$ and determines the relations among the set of the parameters $\left\{x_{r}\right\}$ and $\left\{\tilde{x}_{r}\right\}$. From the canonical commutation relation $\left[\widetilde{Q}_{0}, \widetilde{P}_{0}\right]=1$, we must have $\partial \tilde{x}_{r} / \partial x=\delta_{r, 1}$. By inspecting the relation (5.14), we see that the first coefficient $u_{0}$ in $U$ can be set to be

$$
u_{0}=\exp -\frac{p+q-1}{p q} x_{p+q-1} x_{1}
$$

3 The problem of interchanging $p$ and $q$ in the $(p, q)$ model has been previously discussed by Kawai [15] using a different method in the Schwinger-Dyson approach 
and that $u_{-1}$ obeys

$$
\begin{aligned}
u_{-1}^{\prime} & +\frac{p+q-1}{p q} x_{p+q-1-1} \\
= & -\left(\frac{1}{2}(p-1) u_{0}^{\prime \prime}+\frac{(p+q-1)(p-1)}{p q} x_{p+q-1} u_{0}^{\prime}+\frac{p+q-2}{p q} x_{p+q-2} u_{0}\right),
\end{aligned}
$$

and similar equations for the higher coefficients. It is straightforward but tedious to recursively solve $u_{i}$ 's from (5.14), and substituting back to (5.15), to determine $\tilde{x}_{r}$ 's in terms of $x_{r}$ 's. Since this is not very illuminating, we only mention a few simple general properties; (1) $u_{-i}$ 's take the form (polynomial of $\left.x_{r}{ }^{\prime} s\right) \times \exp \left(-(p+q-1) x_{p+q-1} x_{1} / p q\right)$ apart from an over-all normalization which is independent of $x_{1}$. (2) In particular, at the critical point where $x_{r}=0$ for $2 \leqq r \leqq p+q-1, u_{-1}=0$. (3) $\tilde{x}_{r}=x_{r}+f_{r}\left(x_{p+q-1}, x_{p+q-2}, \ldots, x_{r+1}\right)$, where $f_{r}$ $(1 \leqq r \leqq p+q-2)$ are polynomials and $f_{p+q-1}=0$.

The canonical transformation $U$ leads to the following transformation of the dressing operator $K$,

$$
K \rightarrow \widetilde{K}=u_{0}^{-1} K U .
$$

In general, the $\tau$-function corresponding to $K$ or $\tilde{K}$ for the non-critical theories (namely, "massive" theories) defined around the $(p, q)$ or $(q, p)$ critical points, respectively, are different. At the critical points, the above property (2) indicates that the $\tau$-functions in both representations are mutually identical as the functions of $x_{1}$, since $K_{-1}=-\partial_{1} \log \tau=\widetilde{K}_{-1}=-\partial_{1} \log \tilde{\tau}$, as it should be. However, the definition of the scaling operators are different according to $(p, q)$ or $(q, p)$ representations. Hence the partition functions are in general different except at the critical points. In particular, in the $(p, q)$ representation, the scaling operators $O_{n q}$ corresponding to the coupling constants $x_{n q}(n=1,2, \ldots)$ do not exist since $\partial_{n q} \tau=0$, while, in the $(q, p)$ representation, the ones $\widetilde{O}_{n p}$ corresponding to $\tilde{x}_{n p}$ do not since $\partial_{n p} \tau=0$. This and property (3) imply that $\widetilde{O}_{n q}\left(O_{n p}\right)$ are composite operators consisting of the products of the operators in the set $\left\{O_{p+q-1}, \ldots, O_{n q+1}\right\}$ $\left(\left\{\widetilde{O}_{p+q-1}, \ldots, \widetilde{O}_{n p+1}\right\}\right)$.

Finally, we note an indication that our dual transformation may indeed be interpreted as a dual transformation in the world-sheed picture. It is well known that the $(p, q)$ minimal conformal models are embedded in a free scalar field theory $^{4}$ (with indefinite metric) compactified in a cirle. In the normalization, $\left\langle\phi\left(z_{1}, \bar{z}_{1}\right) \phi\left(z_{2}, \bar{z}_{2}\right)\right\rangle \sim-2 \log \left|z_{1}-z_{2}\right|$, the radius of the circle is $R=2 \sqrt{q / p}$. Note that here $z, \bar{z}$ are the world-sheet coordinates which are nothing to do with $k$. The primary states with conformal weights $h_{r s}=\left((r p-s q)^{2}-(p-q)^{2}\right) / 4 p q(1 \leqq r \leqq q-1$, $1 \leqq s \leqq p-1)$ correspond to the insertion of external lines with zero-mode momentum $r$ (in the unit of $R^{-1}$ ) and winding number $s$. The left (right) moving momentum is given by $p_{0}=\frac{r}{R}+\frac{s R}{2}\left(\bar{p}_{0}=\frac{r}{R}-\frac{s R}{2}\right)$. Thus the interchange $p \leftrightarrow q$ induces transformations $R \leftrightarrow 2 / R$ and $(r, s) \leftrightarrow(s, r)$, namely, the interchange of the roles of momentum and winding number. It is an interesting problem to find a direct connection between these dual transformations. To achieve this, we need more precise knowledge about the nature of the free-field representation in $2 \mathrm{D}$ gravity.

${ }^{4}$ See, e.g., [16] and the references therein 


\section{Concluding Remarks}

In this work, we have tried to develop a canonical formalism for non-perturbative $2 \mathrm{D}$ gravity based on the action principle. We believe that this formulation clarifies some geometrical aspects of $2 \mathrm{D}$ gravity.

The formulation is only concerned about the invariants, such as the cosmological constant and its conjugate, without directly treating the field operators on the 2D world sheets. In this sense, what we have presented is a sort of $S$-matrix theory for 2D gravity. Although such seems more or less inevitable in the situation with the existence of strong topology fluctuation, an important question is then how to transform back and forth to the world-sheet picture. In particular, a direct derivation of the classical canonical structure which describes the genus-zero surfaces, starting from the world sheet theory such as the Liouville theory coupled with minimal conformal models [17, 18] or the topological gravity [19], is desirable. It will be useful to clarify the physical significance of the constraint in the action principle which is essentially an analyticity requirement with respect to the puncture eigenvalue $k$.

Besides this problem, the most urgent question is whether the systems with $c \geqq 1$ matter systems can be incorporated into the present framework. Since the notion of the phase space consisting of the cosmological constant and its conjugate is independent of the target space of the matter fields, the question is reduced to the existence of the generalized momentum and coordinate corresponding to such systems. This seems plausible at least for the $c=1$ model. Even for $c \geqq 1$, a characteristic feature of the action principle, the "spontaneous" emergence of the coupling constants out of the phase space of the cosmological constant and its conjugate, might survive.

Acknowledgements. The author would like to thank the participants of Komaba Seminars and Hokkaido Winter School (Jan., 1991), where the main part of this work was presented, for stimulating discussions. This work is supported in part by a Grant-in-Aid for Scientific Research from the Ministry of Education, Science and Culture, No. 63540207.

\section{References}

1. Brézin, E., Kazakov, V.A.: Phys. Lett. 236 B, 144 (1990);

Douglas, M., Shenker, S.H.: Nucl. Phys. B 335, 635 (1990);

Gross, D.J., Migdal, A.A.: Phys. Rev. Lett. 64, 127 (1990)

2. Douglas, M.R.: Phys. Lett. 237 B, 43 (1990)

3. Jevicki, A., Yoneya, T.: Mod. Phys. Lett. A 5, 1615 (1990)

4. Yoneya, T.: Univ. of Tokyo (Komaba) preprint UT-Komaba 90-28, to appear in Int. Journ. Mod. Phys. A; See also Yoneya, T., to be published in: Proc. of Symposium "Quarks, Symmetries, and Strings" (October, New York, 1990). Jevicki, A., Kaku, M., Kikkawa, K. (eds.). Singapore: World Scientific

5. Fukuma, M., Kawai, H., Nakayama, R.: Univ. of Tokyo-KEK preprint UT-562, KEK-TH251 (1990)

Dijkgraaf, R., Verlinde, E., Verlinde, H.: Princeton preprint IASSNS-HEP-90/48 (1990)

6. Fukuma, M., Kawai, H., Nakayama, R.: Univ. of Tokyo-KEK preprint UT-572, KEK-TH$272(1990)$

7. Adler, M.: Invent. Math. 50, 219 (1979)

8. Ginsparg, P., Goulian, M., Plesser, M.R., Zinn-Justin, J.: Nucl. Phys. B 342, 539 (1990)

9. Brézin, E., Itzykson, C., Parisi, G., Zuber, J.B.: Commun. Math. Phys. 59, 35 (1978) 
10. Bakas, I.: Phys. Lett. B 228, 57 (1989)

11. Pope, C.M., Romans, L.J., Shen, X.: Phys. Lett. B 242, 401 (1990)

12. Date, E., Jimbo, M., Kashiwara, M., Miwa, T.: In: Proc. of RIMS Symposium on Non-Linear Integrable Systems. Jimbo, M., Miwa, T. (eds.). Singapore: World Scientific 1983

13. Moore, G.: Commun. Math. Phys. 133, 261 (1990)

14. Nishigaki, S., Yoneya, T.: Nucl. Phys. B 348, 787 (1991)

15. Kawai, H.: Talk at the Komaba Seminars (Jan., 1991)

16. Felder, G.: Nucl. Phys. B 317, 215 (1989)

17. Knizhnik, V.G., Polyakov, A.M., Zamolodchikov, A.B.: Mod. Phys. Lett. A 3, 819 (1988)

18. David, F.: Mod. Phys. Lett. A 3, 1615 (1988)

Distler, J., Kawai, H.: Nucl. Phys. B 342, 501 (1989)

19. Witten, E.: Nucl. Phys. B 340, 281 (1990)

Distler, J.: Nucl. Phys. B 342, 523 (1990)

Li, K.: Caltech preprint CALT-68-1662, 1670 (1990)

Dijkgraaf, R., Verlinde, H., Verlinde, E.: Princeton preprint PUPT-1204 (1990)

Communicated by N. Yu. Reshetikhin 
